\title{
ÍNDICES AGRONÔMICOS DO MELOEIRO ASSOCIADOS À DOSE ADEQUADA DE NITROGÊNIO, EM AMBIENTE PROTEGIDO E NO CAMPO
}

\author{
Muskmelon plant agronomical indice values associated with \\ adequadenitrogen fertilizer rate, in unheated greenhouse and field
}

\author{
Evando Luiz Coelho', Paulo Cezar Rezende Fontes ${ }^{2}$
}

\begin{abstract}
RESUMO
Objetivou-se com este trabalho determinar a magnitude dos índices agronômicos da folha do meloeiro (Cucumis melo L.), representados pela área (AFQ) e massa da matéria seca da quinta folha à partir do ápice (MSQ), associados à dose de nitrogênio para a produção comercial máxima de frutos (PMF), em duas épocas de amostragens e em dois ambientes. Dois experimentos, ambiente protegido e campo, foram conduzidos adotando-se procedimentos similares. Cada experimento constou de quatro blocos ao acaso contendo cinco tratamentos que foram cinco doses de nitrogênio $\left(0,75,150,300\right.$ e $\left.450 \mathrm{~kg} \mathrm{ha}^{-1} \mathrm{de} \mathrm{N}\right)$, como uréia. Parte desse foi colocado nos sulcos (30\%), antes do transplante e os $70 \%$ restantes foram aplicados via água de irrigação por gotejamento durante $o$ ciclo da cultura. Inicialmente, aos 14 dias após o transplante (DAT), foi determinada a intensidade da clorose nas folhas por meio de notas de 0 (nenhuma clorose) a 5 (clorose acentuada). Aos 25 e 53 DAT, no pegamento do primeiro e do segundo fruto, a AFQ e o MSQ foram determinados. Em ambos os experimentos, a intensidade da clorose diminuiu e AFQ e MSQ aumentaram com o aumento da dose de nitrogênio. Os valores da intensidade da clorose associados à PMF foram 0,22 e 1,11 no ambiente protegido e no campo, respectivamente. Na primeira e segunda amostragens, no ambiente protegido, os valores de AFQ associados à PMF foram $107 \mathrm{e} 72 \mathrm{~cm}^{2}$ e os de PSQ foram 0,36 e 0,26 g, respectivamente. No campo, os valores correspondentes foram 89 e $69 \mathrm{~cm}^{2}$ e 0,39 e $0,30 \mathrm{~g}$, respectivamente. Esses valores são propostos como índices do meloeiro indicadores da adequada dose do fertilizante nitrogenado.
\end{abstract}

Termos para indexação: Cucumis melo, melão, diagnóstico, área foliar, massa da folha seca.

\begin{abstract}
The objective of the experiments was to determine the values of the agronomical melon (Cucumis melo L.) leaf indices, represented by both area (FLA) and dry weight (FLW) of the fifth leaf from the apex associated with the nitrogen rate for the maximum melon fruit yield (MFY), at two samples dates and two environmental conditions. Two experiments, in unheated greenhouse and field conditions, were carried out with similar procedures. Each experiment consisted of four randomized blocks containing five treatments or five nitrogen rates $\left(0,75,150,300\right.$, and $450 \mathrm{~kg} \mathrm{ha}^{-1}$ of $\left.\mathrm{N}\right)$, applied as urea. Part of the fertilizer was placed in furrows $(30 \%)$, before seedling transplantation, and the remaining $70 \%$ were trickle-applied through part of the plant cycle. Initially, 14 days after transplantation, plant chlorosis intensity was evaluated by scores from 0 (no chlorosis) to 5 (leaves completely chlorotics). At 25 and 53 days after transplantation, at first and second fruit setting, FLA and FLW were determined. At both experiments, chlorosis intensity decreased, FLA and FLW increased with increasing $\mathrm{N}$ rates. Chlorosis intensity values associated with MFY were 0.22 and 1.11 at greenhouse and field, respectively. In the greenhouse, at first and second sampling dates, FLA values associated with MFY were 107 and $72 \mathrm{~cm}^{2}$ and FLW values were 0.36 and $0.26 \mathrm{~g}$, respectively. In the field, the corresponding values were 89 and $69 \mathrm{~cm}^{2}$ and 0.39 and $0.30 \mathrm{~g}$, respectively. These are proposed melon plant index values indicators of the added $\mathrm{N}$ fertilizer rate adequacy.
\end{abstract}

Index terms: Cucumis melo, cantaloupe, diagnostic, leaf area, leaf dry weight.

(Recebido para publicação em 19 de julho de 2004 e aprovado em 10 de março de 2005)

\section{INTRODUÇÃO}

Na região sudeste, para produzir fruto de melão rendilhado, Cucumis melo L., grupo cantalupensis, de elevado padrão comercial, pode ser necessário a utilização de ambiente protegido, principalmente em determinadas épocas do ano. Nessa região, períodos chuvosos durante a maturação pioram a qualidade visual do fruto e favorecem o aparecimento de patógenos na planta. Como resultado, há formação de fruto pequeno, com deficiente reticulação, queimado, com baixo teor de açúcar, com qualidade inferior e não mantendo um padrão.
Em ambiente protegido, é comum a aplicação de doses supra-ótimas de nitrogênio resultando em crescimento vegetativo excessivo e redução da produção comercial de frutos de melão (COELHO, 2001), além de aumentar a probabilidade de salinização do solo. Aumentando-se a dose de nitrogênio $(\mathrm{N})$, até determinado limite, haverá quase sempre, mudanças em várias características do meloeiro como acréscimos na área foliar, na massa da folha, na intensidade da cor verde das folhas (COELHO et al., 2002), no número de flores e de frutos por planta, refletindo na massa média e na produção de frutos de melão (BRANTLEY \& WARREN, 1961; FARIA et al., 2000; FLOCKER et al., 1965; TYLER \& LORENZ, 1964).

${ }^{1}$ Engenheiro Agrônomo, MS - Departamento de Fitotecnia/UFV - 36.571-000 - Viçosa, MG.

2 Professor Titular do Departamento de Fitotecnia/UFV - 36571-000 - Viçosa, MG. Bolsista do CNPq - pacerefo@ufv.br 
Portanto, é necessário manejar corretamente a fertilização nitrogenada na cultura. $\mathrm{O}$ ajuste no programa de fertilização nitrogenada pode ser conseguido por correto monitoramento e diagnóstico do estado nutricional das plantas (COLTMAN, 1988; HUETT et al., 1997). Tal procedimento envolve, tradicionalmente, a análise do teor de $\mathrm{N}$ na planta e posterior interpretação do resultado, utilizando-se, quase sempre, o critério do nível crítico (FONTES, 2001). Entretanto, esse tipo de análise pode ter uso limitado, devido ao tempo gasto entre a amostragem e a obtenção dos resultados, além da necessidade de utilização de mão-de-obra, aparelhos e laboratório especializados que tornam o custo da análise elevado. Atualmente, o sensoriamento em tempo real do estado nutricional nitrogenado das plantas tem despertado mais interesse devido às avaliações serem mais rápidas, fáceis e de baixo custo.

Em analogia ao uso do critério de nível crítico de $\mathrm{N}$, acredita-se ser possível estabelecer um nível crítico agronômico, baseado em determinada característica da planta sensível à aplicação de N (FONTES, 2001). A correlação entre determinada característica da planta e a reflexão da luz no dossel e desta com o estado nutricional em $\mathrm{N}$ tem sido investigada como parte da agricultura de precisão, utilizando-se o sensoriamento remoto (BLACKMER et al., 1996; VOUILLOT et al., 1998).

A característica escolhida pode ser usada como índice indireto para o diagnóstico do estado nutricional em nitrogênio (RONCHI et al., 2001) e para avaliar a capacidade produtiva da planta em condições em que o $\mathrm{N}$ é fator limitante. A possibilidade de se utilizar um índice da planta como indicador da adequação da dose do fertilizante nitrogenado não tem sido explorada. Normalmente, a dose de $\mathrm{N}$ ainda tem sido estabelecida empiricamente, ao contrário dos demais macronutrientes, cuja limitação pode ser prevista pela análise do solo.

São escassas as informações na literatura sobre possível relação entre a dose adequada de $\mathrm{N}$ e o correspondente valor de determinada característica de crescimento do meloeiro. Esse valor, possível de ser chamado de índice agronômico ou fitotécnico, poderá ser uma ferramenta indicadora, com rapidez e certa precisão, no acerto na dose recomendada de fertilizante nitrogenado, podendo ser utilizada por técnicos, no campo, desde que apropriadamente calibrada.

Para a utilização do índice agronômico, é necessário o estabelecimento de um valor para ser usado como padrão de comparação ou "nível crítico". O presente trabalho teve como objetivo determinar o "valor crítico" de índices agronômicos, em duas épocas de amostragens, para o meloeiro cultivado no campo e em ambiente protegido.

\section{MATERIAL E MÉTODOS}

Dois experimentos foram conduzidos na Universidade Federal de Viçosa, na Horta de Pesquisa do Departamento de Fitotecnia, situada a $693 \mathrm{~m}$ de altitude, $20^{\circ} 45^{\prime}$ sul e $42^{\circ} 51^{\prime}$ 'Oeste, clima do tipo CWA, no período de dezembro a março (1997/98). Nesse período, a precipitação foi $497 \mathrm{~mm}$, a evaporação 275 $\mathrm{mm}$, a insolação 593 horas, a temperatura média $24,0^{\circ} \mathrm{C}$ e a umidade relativa $78 \%$.

O primeiro experimento foi realizado no campo sem proteção plástica e o segundo, em ambiente protegido. A cobertura seguiu o modelo capela, com largura de $9 \mathrm{~m}$, comprimento de $40 \mathrm{~m}$, altura dos esteios laterais de $3 \mathrm{~m}$ e a altura do vão central de $3,8 \mathrm{~m}$, com frontais e laterais fechadas com filme de polietileno transparente de $0,1 \mathrm{~mm}$ de espessura.

Os experimentos foram conduzidos de forma similar. O delineamento experimental foi o de blocos casualizados, com quatro repetições. Os tratamentos consistiram de cinco doses de nitrogênio $(0,75,150,300$ e $450 \mathrm{~kg} \mathrm{ha}^{-1} \mathrm{de} \mathrm{N}$ ), na forma de uréia, sendo $30 \%$ das doses colocadas nos sulcos, antes do transplante das mudas e os $70 \%$ restantes aplicadas via água de irrigação, por gotejamento. A parcela possuía 4,0 m de largura por 4,5 m de comprimento, sendo constituída de quatro fileiras, no espaçamento de $1 \mathrm{~m}$ entre cada uma. $\mathrm{O}$ espaçamento entre plantas nas fileiras foi $0,45 \mathrm{~m}$. Como parcela útil foram consideradas as duas fileiras internas, cada uma com oito plantas.

Utilizou-se o híbrido Trusty, da Sun Seed, que apresenta frutos ovais, com suturas na casca, polpa salmão, pequena cavidade interna, apresentando massa média em torno de $1000 \mathrm{~g}$. A semeadura foi realizada em bandejas de poliestireno expandido com 128 células, em 03/12. O substrato foi preparado com sub-solo, terriço (solo do horizonte A sob vegetação de mata) e de palha de arroz carbonizada em proporções iguais. A mistura foi adubada com $0,2 \mathrm{~kg}$ de superfosfato simples e $3 \mathrm{~kg}$ de calcário dolomítico por metro cúbico.

O solo dos dois ambientes foi classificado como Argissolo Vermelho-Amarelo Câmbico, fase terraço. Antes do preparo, o solo do ambiente protegido recebeu quantidade excessiva de água, que ficou represada por trinta dias, visando à eliminação de excesso de sais, 
principalmente os nitrogenados. Em seguida, o solo de ambos os experimentos receberam calcário dolomítico para elevar a saturação por bases a 70\%, fazendo-se sua incorporação por aração profunda e duas gradagens. Dois meses após a calagem, o solo foi arado, gradeado e sulcado. No sulco de plantio, efetuou-se a adubação, expressa em kg ha-1, com 150 de $\mathrm{P}_{2} \mathrm{O}_{5}$, na forma de superfosfato simples; 45 de $\mathrm{K}_{2} \mathrm{O}$, na forma de cloreto de potássio; 200 de sulfato de magnésio; 10 de bórax, 10 de sulfato de zinco e 0,2 de molibdato de amônio, além de 30 $\%$ da correspondente dose de nitrogênio na forma de uréia. Uma semana após a adição dos fertilizantes, em 19/ 12, em solo úmido, foi feito o transplante. Foram utilizadas mudas no estádio da terceira folha completamente desenvolvida.

A irrigação foi por gotejamento com os emissores espaçados de $0,30 \mathrm{~m}$. O turno de rega foi diário e determinado pela evapotranspiração da cultura, baseado em tanque classe A. Quinze dias após o transplante foi iniciada a fertirrigação, sendo que as doses de $\mathrm{N}$ (tratamentos) e a dose de $\mathrm{K}_{2} \mathrm{O}$ (105 kg ha-1), como $\mathrm{KCl}$, para todos os tratamentos, foram fornecidas em sete aplicações, em intervalos semanais. Em cada uma das duas primeiras aplicações foram aplicados $8 \%$ do $\mathrm{N}$ e $10 \%$ do $\mathrm{K}_{2} \mathrm{O}$. Nas três seguintes, $23 \%$ do N e $20 \%$ do $\mathrm{K}_{2} \mathrm{O}$ e, finalizando, nas duas últimas, $8 \%$ do $\mathrm{N}$ e $10 \%$ do $\mathrm{K}_{2} \mathrm{O}$.

As plantas foram conduzidas verticalmente sem poda de ramos ou raleio de frutos e tutoradas em espaldeira de bambu disposto horizontalmente ao solo e fixado em mourões fincados no solo. O controle fitossanitário e os demais tratos culturais foram realizados sempre que necessários. Foram realizadas três colheitas, com intervalos de cinco dias, obtendo-se a produção comercial de frutos (dados não apresentados), sendo considerados comerciais os frutos firmes, uniformes na cor, com perfume almiscarado característico, desprovidos de danos físicos e com o diâmetro entre 9 a $20 \mathrm{~cm}$.

Aos 14 dias após o transplante, a intensidade da clorose ou índice de clorose (IC) foi determinado por notas de 0 a 5 atribuídas às plantas. A nota 0 foi atribuída à planta com as folhas cotiledonares (FC) e $1^{\text {a }}$ folha definitiva (FD) de cor verde escuro; a nota 1 , às plantas com as FC e FD de cor verde; a nota 2, às plantas com as FC clorótica e FD de cor verde; a nota 3 , às plantas com as FC clorótica e FD parcialmente clorótica; a nota 4, às plantas com as FC e FD totalmente clorótica e a nota 5 , às plantas com as FC necrosada e FD totalmente clorótica.

Também, foi determinada a área foliar, com o aparelho Li-3100. As medições foram realizadas na quinta folha a partir do ápice do ramo principal (AFQ), em duas épocas. A primeira, no período compreendido entre o florescimento e o pegamento do $1^{\circ}$ fruto e a segunda, no período compreendido entre o florescimento e o pegamento do $2^{\circ}$ fruto. Também, naquelas mesmas datas de amostragens, foi avaliada a massa da matéria seca da quinta folha a partir do ápice do ramo principal (PSQ), após a folha ter sido secada em estufa a $70^{\circ} \mathrm{C}$, até massa constante.

Os resultados foram interpretados por análises de variância e de regressão, tendo sido testados o efeito linear, quadrático e cúbico do modelo polinomial $\mathrm{y}=\mathrm{a}+\mathrm{bx}+\mathrm{b}_{1} \mathrm{x}^{2}$ $+b_{2} x^{3}$ e a raiz quadrada do modelo polinomial, $y=a+b x^{0,5}+$ $b_{1} x+b_{2} x^{1,5}$. Foi selecionada, dentro de cada grupo de equações com o mesmo número de parâmetros estimados, aquela de efeito significativo pelo teste $\mathrm{F}$ a $5 \%$ de probabilidade e de maior soma de quadrados. A partir da derivada primeira da equação ajustada, foi determinada a dose de $\mathrm{N}$ necessária para ser atingida a produção comercial máxima de frutos (PMF). Tais doses foram $312 \mathrm{e}$ $344 \mathrm{~kg} \mathrm{ha}^{-1}$ de $\mathrm{N}$, na estufa e no campo, que propiciaram as produtividades de 30,01 e $29,25 \mathrm{t} \mathrm{ha}^{-1}$, respectivamente (COELHO, 2001).

Para cada época de amostragem e ambiente foram calculados os valores dos índices agronômicos, AFQ e PSQ, associados à dose de nitrogênio para a produção comercial máxima de frutos (PMF). Para tal, foram utilizados os valores de 312 e $344 \mathrm{~kg} \mathrm{ha}^{-1}$ de $\mathrm{N}$ na equação obtida para cada característica, seguindo-se a metodologia mostrada em Fontes (2001).

\section{RESULTADOS E DISCUSSÃO}

Nos dois ambientes, na avaliação realizada 14 dias após o transplante, as plantas deficientes em $\mathrm{N}$ apresentaram clorose nas folhas cotiledonares e primeira definitiva sendo a intensidade inversamente influenciada pelas doses de N (Figuras 1a e 1b). Na estufa, o valor da intensidade da clorose associada à dose de nitrogênio para a produção comercial máxima de frutos (PMF) foi 0,22; no campo, o valor correspondente foi 1,11. Correlações significativas entre a intensidade da cor verde com a concentração $\mathrm{N}$ nas folhas tem sido encontrada por diversos autores (CHAPMAN \& BARRETO, 1997; GUIMARÃES et al., 1999; SEXTON \& CARROLL, 2002), pois grande parte do $\mathrm{N}$ contido nas folhas participa da síntese e da estrutura das moléculas de clorofila (TAIZ \& ZEIGER, 2004).

Ciênc. agrotec., Lavras, v. 29, n. 5, p. 974-979, set./out., 2005 
Tanto a folha cotiledonar quanto a primeira folha definitiva do meloeiro que recebeu a dose ótima de $\mathrm{N}$ apresentavam coloração verde, sem clorose, indicando que a intensidade da clorose ou do verde, avaliada em determinado período do estádio de crescimento da planta, pode ser ferramenta na diagnose do estado nutricional de nitrogênio da planta. A clorose das folhas do meloeiro é o sintoma clássico e de relativamente fácil percepção da deficiência de N, conforme observado por Belfort (1985). Além da clorose, em caso de deficiência de $\mathrm{N}$ há a formação de folhas pequenas, retardamento do crescimento e decréscimo no número e peso dos frutos formados (BELFORT et al., 1986; NERSON et al., 1987). Aqueles sintomas e redução da produção do meloeiro também foram observados em ambos os experimentos.

Em ambos os experimentos, nas avaliações realizadas no pegamento do 1ㅇroto, a AFQ aumentou com o aumento da dose de $\mathrm{N}$ (Figuras $2 \mathrm{a}$ e $2 \mathrm{~b}$ ). O efeito positivo do $\mathrm{N}$ no crescimento da folha está de acordo com os resultados encontrados por Nerson (1992) que descreveu a influência positiva da adubação nitrogenada no crescimento vegetativo, principalmente do limbo foliar, das mudas de melão Galia. Também, no pegamento do 2o fruto, a AFQ aumentou com o aumento das doses de $\mathrm{N}$.

No ambiente protegido, nas amostragens realizadas por ocasião do pegamento do $1^{\circ}$ e do $2^{\circ}$ fruto, os valores da AFQ associados com a dose de $\mathrm{N}$ que propiciou a PMF foram 107,18 e $71,77 \mathrm{~cm}^{2}$, respectivamente. No campo, nas

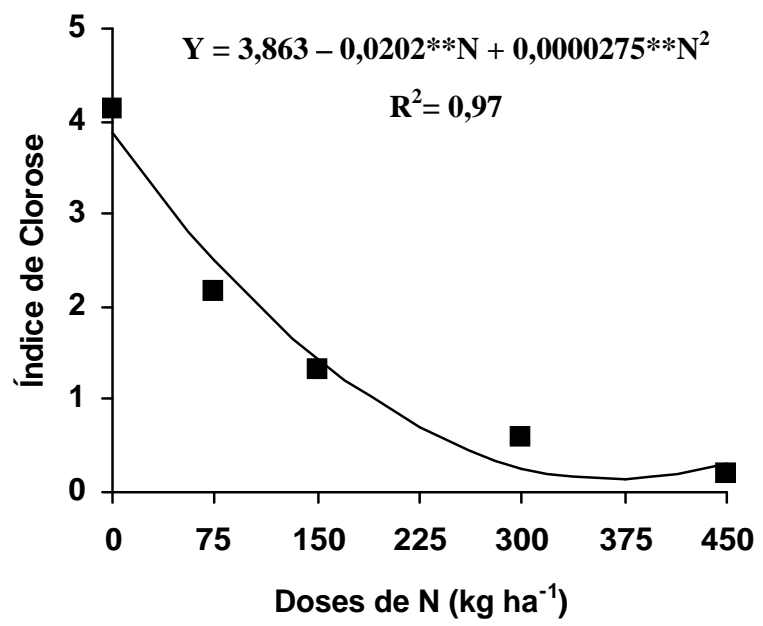

duas ocasiões, os valores correspondentes foram 88,91 e $69,01 \mathrm{~cm}^{2}$, respectivamente. Os valores de AFQ foram, aparentemente, menores na segunda avaliação que na primeira, devido, talvez, à maior competição por fotoassimilados, direcionados preferencialmente para o desenvolvimento dos frutos. A aparente maior área foliar das plantas no ambiente protegido pode ter sido a resposta das plantas ao decréscimo de energia incidente causada pelo plástico de cobertura forçando o desenvolvimento de maior área da folha para compensar a produção fotossintética. No campo, no período de 19 a 50 dias após a semeadura foi encontrada variação de 50 a $143 \mathrm{~cm}^{2}$ para a área de todas as folhas do meloeiro Amarelo.

Em ambos os ambientes, tanto no pegamento do $1^{\circ}$ quanto do $2^{\circ}$ fruto, houve aumento do PSQ com o aumento da dose de N (Figuras 3a e 3b). Na estufa, no pegamento do $1^{\circ}$ e e do $2^{\circ}$ fruto, com a dose de $\mathrm{N}$ que propiciou a PMF, os valores de PSQ foram 0,36 e 0,26 g, respectivamente. Os valores correspondentes, em plantas no campo, foram 0,39 e $0,30 \mathrm{~g}$, respectivamente. Tais valores podem ser considerados ou chamados de "críticos". Não foram encontrados referências a valores de características agronômicas do meloeiro associados à dose ótima de nitrogênio. Aparentemente, os valores "críticos" de AFQ e de PSQ diminuíram na segunda amostragem em relação a primeira, quando a planta estava na fase de crescimento do primeiro e no aparecimento do segundo fruto, indicando a força do dreno.

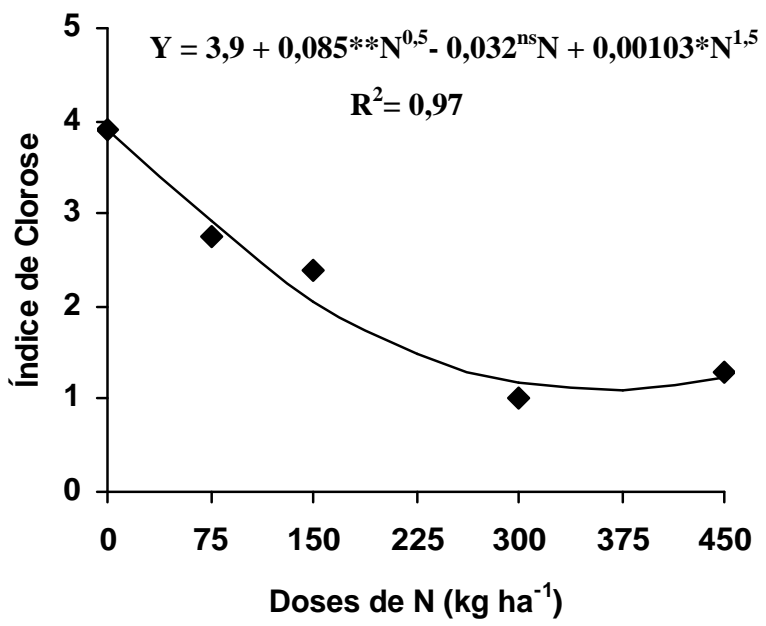

FIGURA 1 - Intensidade de clorose das folhas do meloeiro, em avaliação realizada 14 dias após o transplante, em função de doses de nitrogênio (N), em estufa (a) e no campo (b). UFV, Viçosa (MG), 2004. 

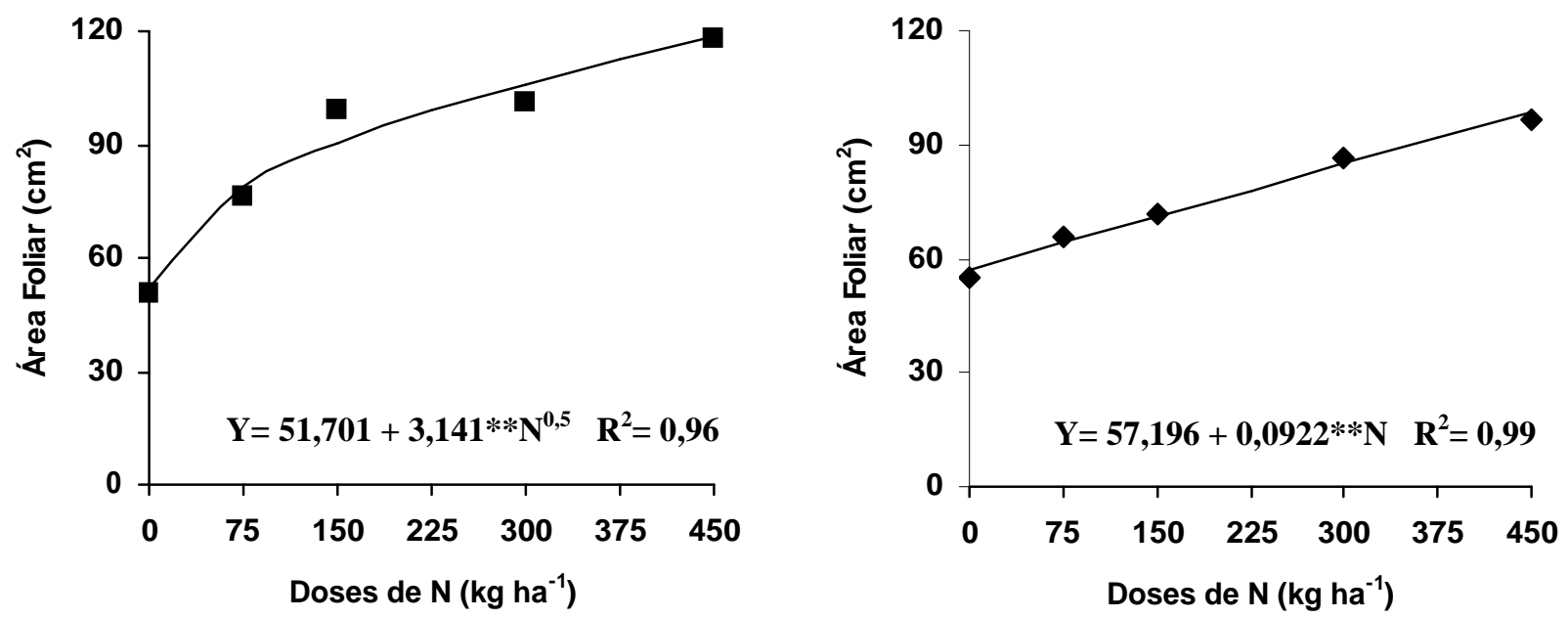

FIGURA 2 - Área da quinta folha totalmente expandida a partir do ápice do meloeiro (AFQ), em avaliação realizada no pegamento do primeiro fruto, em função de doses de nitrogênio (N), em estufa (a) e no campo (b). UFV, Viçosa (MG), 2004.
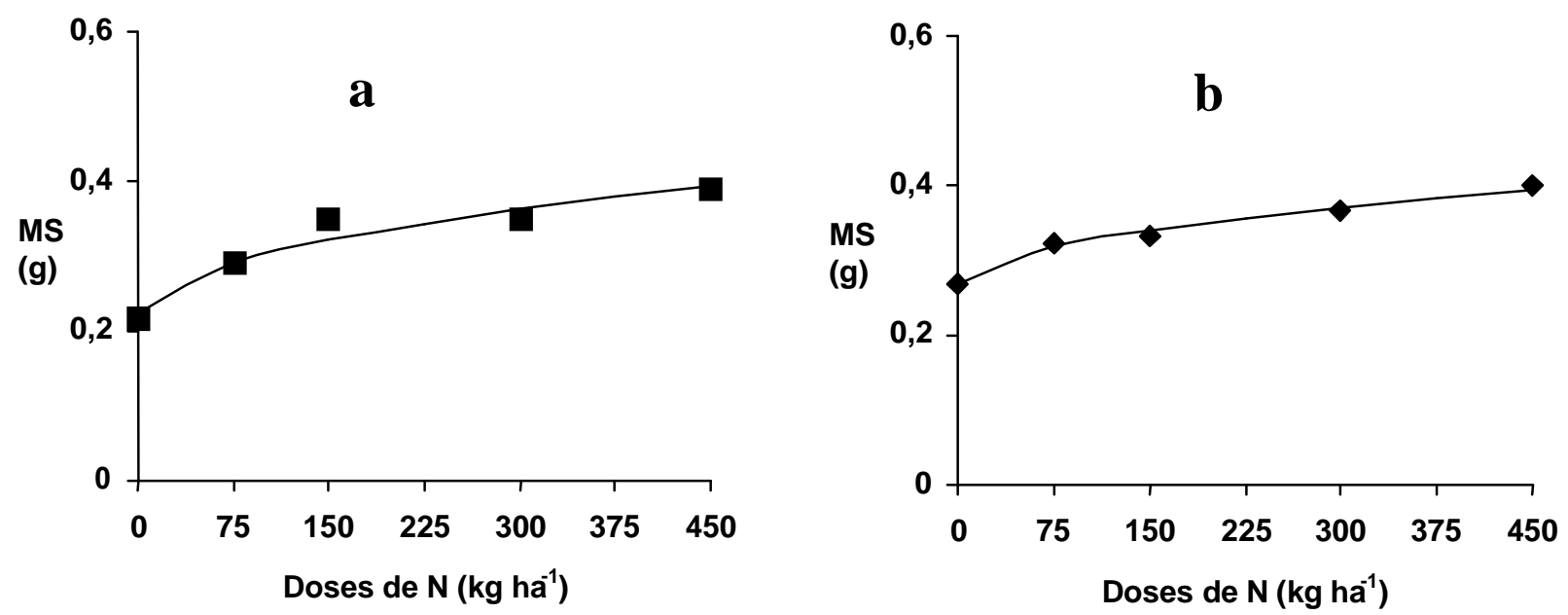

FIGURA 3 - Matéria seca da quinta folha totalmente expandida a partir do ápice do meloeiro (MS) em determinação feita no pegamento do primeiro fruto, em função de doses de nitrogênio, em estufa (a) e no campo (b). UFV, Viçosa (MG), 2004.

\section{CONCLUSÕES}

a) No ambiente protegido e nas amostragens realizadas no pegamento do primeiro e do segundo fruto, a área foliar e a massa da matéria seca da quinta folha a partir do ápice do ramo principal devem ser 107 e $72 \mathrm{~cm}^{2}$, e 0,36 e $0,26 \mathrm{~g}$, respectivamente.

b) No campo e nas amostragens realizadas no pegamento do primeiro e do segundo fruto, a área foliar e a massa da matéria seca da quinta folha a partir do ápice do ramo principal devem ser 89 e $69 \mathrm{~cm}^{2}$, e 0,39 e $0,30 \mathrm{~g}$, respectivamente.

c) Os índices agronômicos precisam ser calibrados para outras condições.

\section{AGRADECIMENTOS}

Ao CNPq pela bolsa de Produtividade em Pesquisa, à FAPEMIG pela bolsa de mestrado e ao estudante de agronomia Janner Siqueira Santana pela ajuda na condução do experimento.

\section{REFERÊNCIAS BIBLIOGRÁFICAS}

BELFORT, C. C. Acumulação de matéria seca e recrutamento de nutrientes em melão (Cucumis melo $\mathrm{L}$. CV. Valenciano Amarelo CAC) cultivado em latossolo vermelho amarelo em Presidente Venceslau -SP. 1985. $72 \mathrm{f}$. Tese (Doutorado) - Escola Superior de Agricultura de Luiz de Queiroz, Piracicaba, 1985. 
BELFORT, C. C.; HAAG, H. P.; MINAMI, K. Nutrição mineral de hortaliças: diagnóstico das carências de macronutrientes e boro em melão (Cucumis melo L.). Anais da ESALQ, Piracicaba, v. 43, n. 1, p. 365-377, 1986.

BLACKMER, T. M.; SCHEPERS, J. S.; VARVEL, G. E.; WALTER-SHEA, E. A. Nitrogen deficiency detection using reflected shortwave radiation from irrigated corn canopies. Agronomy Journal, Madison, v. 88, p. 1-5, 1996.

BRANTLEY, B. B.; WARREN, G. F. Effects of nitrogen nutrion on flowering, fruiting and quality in the muskmelon. Journal of the American Society for Horticultural Science, Alexandria, v. 77, n. 1, p. 424-431, 1961.

CHAPMAN, S. C.; BARRETO, H. J. Using a chlorophyll meter to estimate specific leaf nitrogen of tropical maize during vegetative growth. Agronomy Journal, Madison, v. 89, p. 557-592, 1997.

COELHO, E. L.Produtividade e qualidade de frutos de melão produzidos na estufa e no campo influenciadas por doses de nitrogênio aplicadas por gotejamento. 2001. $79 \mathrm{f}$. Dissertação (Mestrado em Fitotecnia) - Universidade Federal de Viçosa, Viçosa, 2001.

COELHO, E. L.; FONTES, P. C. R.; CARDOSO, A. A.; FINGER, F. L. Nível crítico do índice SPAD na folha do meloeiro na estufa e no campo. In: CONGRESSO BRASILEIRO DE OLERICULTURA, 42., 2002, Brasília, DF. Horticultura Brasileira, Brasília, DF, v. 20, n. 2, jul. 2002. Suplemento 2.

COLTMAN, R. R. Yields of greenhouse tomatoes managed to maintain specific petiole sap nitrate level. HortScience, Alexandria, v. 23, n. 1, p. 148-151, 1988.

FARIA, C. M. B.; COSTA, N. D.; PINTO, J. M.; BRITO, L. T. L.; SOARES, J. M. Níveis de nitrogênio por fertirrigação e densidade de plantio na cultura do melão em um vertissolo. Pesquisa Agropecuária Brasileira, Brasília, v. 35, n. 3, p. 491-495, 2000.

FLOCKER, W. J.; LINGLE, J. C.; DAVIS, R. M.; MILLER, R. J. Influence of irrigation and nitrogen fertilization on yield, quality and size of cantaloupes. Journal of the American Society for Horticultural Science, Alexandria, v. 86, n. 1, p. 424-431, 1965.
FONTES, P. C. R. Diagnóstico do estado nutricional de plantas. Viçosa: UFV, 2001. $122 \mathrm{p}$.

GUIMARÃES, T. G.; FONTES, P. C. R.; PEREIRA, P. R. G.; ALVAREZ, V. H.; MONNERAT, P. H. Teores de clorofila determinados por medidor portátil e sua relação com formas de nitrogênio em folhas de tomateiro cultivados em dois tipos de solo. Bragantia, Campinas, v. 58, n. 1, p. 209-216, 1999.

HUETT, D. O.; MAIER, N. A.; SPARROW, L. A.; PIGGOTT, T. J. Vegetables. In: REUTER, D. J.; ROBINSON, J. B. (Eds.). Plant analysis: an interpretation manual. 2. ed. Collingwood: CSIRO, 1997. p. 385-464.

NERSON, H. Effects of soil type, mineral nutrition and salinity on greenhouse grown muskmelons in winter. Journal of Plant Nutrition, Monticello, v. 15, n. 11, p. 23812403, 1992.

NERSON, H.; PARIS, H. S.; EDELSTEIN, M. Nitrogen and phosphorus stress repair muskmelon (Cucumis melo) seedlings. Journal of Plant Nutrition, Monticello, v. 10, n. 9, p. 1835-1841, 1987.

RONCHI, C. P.; FONTES, P. C. R.; PEREIRA, P. R. G.; NUNES, J. C. S.; MARTINEZ, H. E. P. Índices de nitrogênio e de crescimento do tomateiro em solo e em solução nutritiva. Revista Ceres, Viçosa, v. 48, n. 278, p. 469-484, 2001.

SEXTON, P.; CARROLL, J. Comparison of SPAD chlorophyll meter readings vs. petiole nitrate concentration in sugarbeet. Journal of Plant Nutrition, Monticello, v. 25, n. 9, p. 1975-1986, 2002.

TAIZ, L.; ZEIGER, E. Fisiologia vegetal. 3. ed. Porto Alegre: Artmed, 2004. 719 p.

TYLER, K. B.; LORENZ, O. A. Nutrient absorption and growth of four muskmelon varieties. Journal of the American Society for Horticultural Science, Alexandria, v. 84, n. 1, p. 364-371, 1964.

VOUILLOT, M. O.; HUET, P.; BOISSARD, P. Early detection of $\mathrm{N}$ deficiency in a wheat crop using physiological and radiometric methods. Agronomie, [S.1.], v. 18, p. 117-130, 1998. 